\title{
Phase Imaging in STEM Allowing for Post-Acquisition Aberration Correction and 3D Optical Sectioning using Ptychography Wigner-Distribution Deconvolution
}

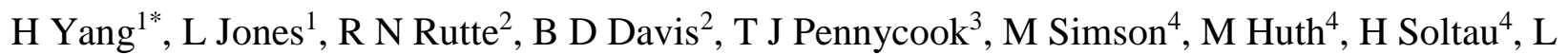 \\ Strüder $^{5}$, R Sagawa ${ }^{6}$, Y Kondo ${ }^{6}$ and P D Nellist ${ }^{1}$ \\ 1. Department of Materials, University of Oxford, Parks Rd, Oxford UK. \\ 2. Department of Chemistry, University of Oxford, 12 Mansfield Road, OX1 3TA, Oxford UK. \\ 3. Department of Physics, University of Vienna, Boltzmanngasse 5, 1090 Vienna, Austria. \\ 4. PNDetector GmbH, Otto-Hahn-Ring 6, 81739 München, Germany. \\ 5. PNSensor GmbH, Otto-Hahn-Ring 6, 81739 München, Germany. \\ 6. JEOL Ltd.,3-1-2 Musashino Akishima Tokyo 196-8558 Japan. \\ * Now at Molecular Foundry, Lawrence Berkeley National Laboratory, Berkeley, CA, 94720, USA.
}

Advances in fast pixelated detector technology have enabled coherent diffraction patterns in a scanning transmission electron microscope (STEM) to be recorded at every probe position to form a four dimensional (4D-) STEM dataset. The rich information contained in the 4D-STEM dataset has previously been poorly utilized by the STEM bright-field detector with a small collection angle, hindering the efficiency for imaging light elements. Furthermore, collecting the electron intensity using integrating detectors such as annular bright field and the annular dark field (ADF) detectors is not able to make full use of the diffraction information.

Electron ptychography is a method for retrieving phase information using diffraction patterns acquired from a sequential array of overlapping illuminated areas, and has been first demonstrated on a 4DSTEM dataset more than 20 years ago for improving image resolution [1]. At that time, the image field of view was restricted by the limitations of the camera speed and computational processing power. Using a modern aberration-corrected STEM and a fast pixelated detector, quantitative phase images can be formed simultaneously with other STEM imaging modes such as ADF at atomic resolution [2]. This phase imaging mode has a monotonic phase contrast transfer function and provides an inherent filter of image noise without reducing the strength of the phase contrast [3].

In this work we show that the large redundancy in phase information in the 4D-STEM data not only offers efficient phase imaging, but also allows lens aberrations to be detected and corrected through post-acquisition processing (Fig.1) by applying a ptychography method called Wigner-distribution deconvolution (WDD) [4]. The aberration measurement and correction method works on both amorphous and crystalline specimens. Moreover, three dimensional structure information is also available post-acquisition by reconstructing the phase image at a specific depth in the specimen along the e-beam direction, which can be performed even though the microscope may not have been focused at that depth (Fig.2). Theoretical calculations suggest that the three dimensional structure information using the WDD method is attributed to an optical sectioning effect, and our experimental results suggest that WDD optical sectioning gives a depth resolution that is equal to the depth of focus of the microscope. [5]

[1] P.D. Nellist, B.C. McCallum and J.M. Rodenburg, Nature 374 (1995) 630-632.

[2] T.J. Pennycook et al., Ultramicroscopy 151 (2015) 160-167.

[3] H. Yang et al., Ultramicroscopy 151 (2015) 232-239. 
[4] J. M. Rodenburg, R. H. T. Bates, Phil. Trans. Roy. Soc. A 339 (1992) 521-553.

[5] The authors acknowledge funding from the EPSRC through grant number EP/M010708/1.

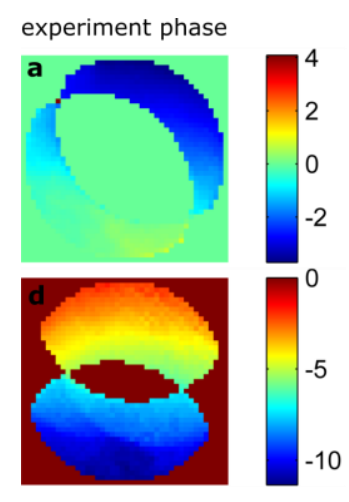

measured phase in condenser aperture

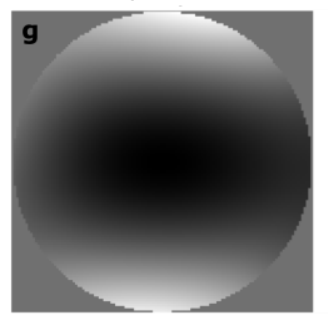

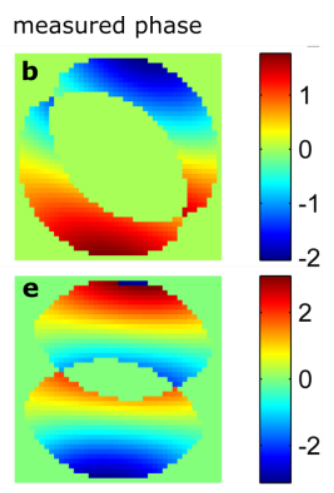
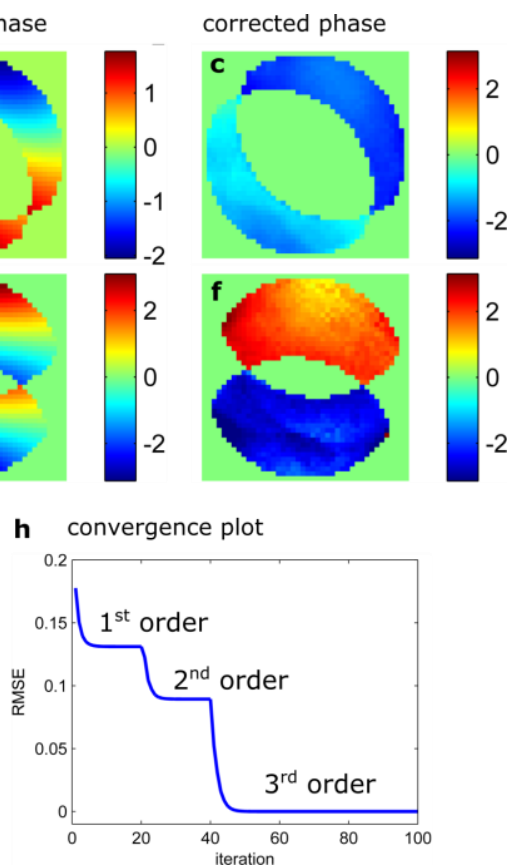

Figure 1. Detection and correction of lens aberrations through post-acquisition processing using WDD. (a) The aberrations result in a non-flat phase surface that is directly measurable experimentally from the ptychographic phase signals, with two examples shown in (a,d). The phase variations due to aberrations can be parameterized and directly measured in $(b, e)$. The experiment phase after being corrected show a reasonably flat phase surface as expected from an aberration free imaging condition in (c, f). The phase inside the probe forming aperture due to the lens aberrations which are measured up to the $3^{\text {rd }}$ order is shown in $(\mathrm{g})$. (h) shows the convergence of the aberration measurement procedure which iteratively measures up to $3^{\text {rd }}$ order aberrations.

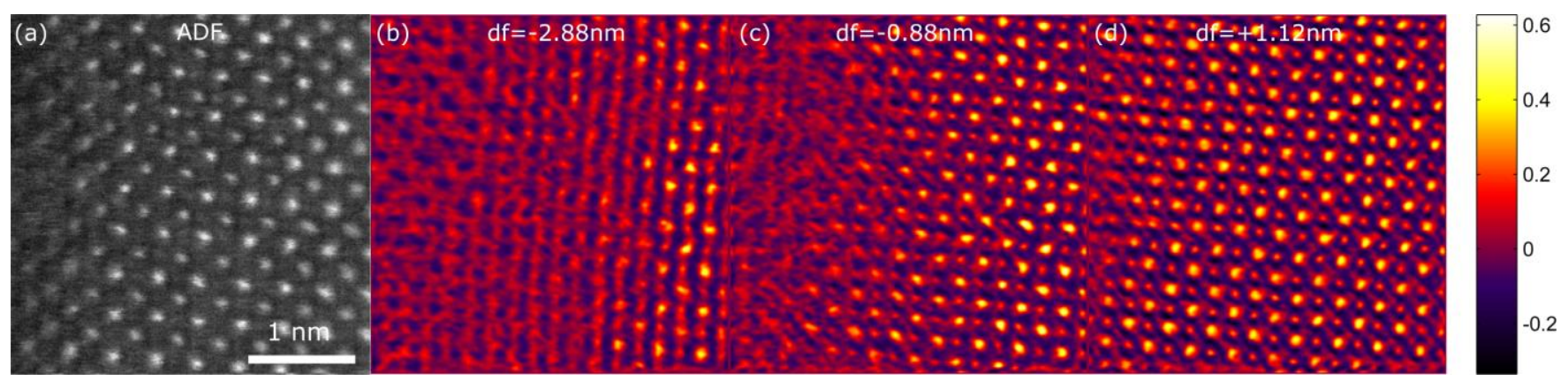

Figure 2. WDD optical sectioning showing the sensitivity of the reconstructed phase to the relative height of the atomic columns of a $\mathrm{SrTiO}_{3}$ wedge sample. (a) Simultaneously recorded ADF image shows the $\mathrm{SrTiO}_{3}$ wedge being slightly out of focus to the left of the image. The atomic columns of relatively different heights are brought to their best focusing condition by varying the defocus aberrations that are deconvolved out of the 4D-STEM dataset, shown in (b-d). Oxygen columns are clearly visible in (b-d) as well. All images are obtained from one 4D-STEM dataset acquired at a single focusing condition. 